〈Subscripts〉

$\begin{array}{ll}1 & =\text { water } \\ 2 & =\text { ethanol } \\ 3 & =\text { minor component } \\ D & =\text { distillate } \\ f & =\text { feed plate } \\ i & =\text { component number, } 2 \text { or } 3\end{array}$
Numerical Method", p. 390, John Wiley \& Sons (1969).

2) Ikari, A., S. Mishima and I. Otsubo: J. Chem. Eng. Japan, 7, 271 (1974).

3) Nihon-kagakukai: "Kagakubinran, Kisohen II", p. 462 (1966).

(Presented at the seminar of Distillation Engineering (Kagoshima) on Nov. 17, 1977.)

\title{
GAS ABSORPTION WITH CHEMICAL REACTION IN CROSSFLOW GAS-LIQUID CONTACTOR WITH CONSIDERATION OF BOTH GAS- AND LIQUID-PHASE RESISTANCES
}

\author{
YoShISHIGE HAYASHI, EIJI HIRAI AND SHUICHI HIGASHI \\ Department of Chemical Engineering, \\ Kanazawa University, Kanazawa 920
}

\begin{abstract}
Theoretical study of crossflow gas absorption accompanied by a second-order irreversible chemical reaction was performed by taking into account both gas- and liquid-phase resistances to absorption and this solution was compared with the results calculated in the previous paper ${ }^{2)}$ on liquid-phase resistance.

Using the theoretical solution proposed in this paper, one can examine how the chemical absorption rate is affected by the change of reactant concentration in the liquid phase or of solute gas composition in the gas phase, and can estimate the concentration profile of the solute gas at the gas-liquid interface.
\end{abstract}

\section{Introduction}

The authors previously reported theoretical and experimental research ${ }^{2,3}$ on the effect of a secondorder irreversible chemical reaction on the rate of gas absorption by the use of a crossflow gas-liquid contactor. In that work, it was assumed that the gasphase resistance to absorption is negligible.

Generally, in the system used in industrial processes, resistance to mass transfer exists in both gas and liquid phases and it is often desirable to determine the solute gas concentration exactly in both the gas and the absorbing liquid.

In this paper, a usable analytical method for gas absorption accompanied by a second-order chemical

Received September 5,1977. Correspondence concerning this article should be addressed to Y. Hayashi. S. Higashi is now with Symco-Foodes Co., Ltd., Osaka 560. reaction is studied by taking into account both gasand liquid-phase resistances.

\section{Theoretical Consideration}

Figure 1 shows a schematic model of a wetted-wall crossflow absorption tower. The liquid flows downward from the top of the tower passing through the cross section $X_{0} Z_{0}$. The gas flows at right angles to $Y_{0} Z_{G 0}$ and comes into contact with the liquid flow at gas-liquid interface, $Z_{G}=Z=0$, in the tower.

In order to solve this problem, some assumptions have been made to reduce the complexity of the equations as follows:- (1) The system is isothermal. (2) Ideal gas law is valid. (3) The flows of gas and liquid are steady and in plug flow. (4) The resistance to mass transfer at the gas-liquid interface is negligible. (5) The chemical reaction occurs only in the liquid layer. (6) The reactant contained in the liquid phase 


\section{1 Physical absorption}

The basic equations in a crossflow absorption tower are given as follows:

$$
\begin{array}{ll}
\frac{\partial \phi_{1}}{\partial \xi}=a_{G} \frac{\partial}{\partial \zeta_{G}}\left\{-\frac{1}{\left(1-y_{A}\right)}-\frac{\partial \phi_{1}}{\partial \zeta_{G}}\right\} & \text { for gas phase } \\
\frac{\partial C_{A 1}}{\partial \eta}=a_{A} \frac{\partial C_{A 1}}{\partial \zeta^{2}} & \text { for liquid phase }
\end{array}
$$

with the boundary conditions

$$
\begin{aligned}
& \text { at } \zeta_{G}=\zeta=0 ; \quad \phi_{1}=1-C_{A 1}, C_{A 1}=f(\xi, \eta), \\
& -\frac{P}{R T} \frac{\mathscr{D}_{G}}{Z_{G 0}} \frac{y_{A 0}-y_{A}^{*}}{1-y_{A}}-\frac{\partial \phi_{1}}{\partial \zeta} \\
& \quad=-\frac{\mathscr{D}_{A}\left(C_{A 0}^{*}-C_{A 0}\right)}{Z_{0}} \frac{\partial C_{A 1}}{\partial \zeta} \\
& \text { at } \xi=0 \quad ; \quad \phi_{1}=0 \\
& \text { at } \eta=0 \quad ; \quad C_{A 1}=0 \\
& \text { at } \zeta_{G \rightarrow \infty} ; \phi_{1}=0 \\
& \text { at } \zeta \rightarrow \infty \quad ; \quad C_{A 1}=0
\end{aligned}
$$

where $a_{A}, a_{G}, \xi, \zeta, \zeta_{G}, \eta, C_{A 1}$ and $\phi_{1}$ are

$$
\begin{aligned}
& a_{A}=\mathscr{D}_{A} Y_{0} / V_{\max } Z_{0}^{2}=\mathscr{D}_{A} t / Z_{0}^{2}, \\
& a_{G}=\mathscr{D}_{G} X_{0} / V_{G} Z_{G 0}^{2}=\mathscr{D}_{G} t_{G} / Z_{G 0}^{2}, \quad \zeta=Z / Z_{0}, \\
& \xi=X / X_{0}, \quad \zeta_{G}=Z_{G} / Z_{G 0}, \quad \eta=Y / Y_{0}, \\
& C_{A 1}=\left(C_{A A}-C_{A 0}\right) /\left(C_{A 0}^{*}-C_{A 0}\right), \\
& \phi_{1}=\left(y_{A 0}-y_{A}\right) /\left(y_{A 0}-y_{A 0}^{*}\right)
\end{aligned}
$$

In this case, it is impossible to solve Eqs. (1) and (2) analytically because $y_{A}$ is a function of $\xi, \eta$ and $\zeta_{G}$. The absorption rate is determined by the concentration gradiant at the gas-liquid interface and, when gas absorption with chemical reaction is studied theoretically, the basic equations in both gas and liquid phases should especially be discussed in detail near the gas-liquid interface.

Now, let us assume that $y_{A}$ in the denominator of the right-hand side in Eq. (1) is replaced by a constant concentration $y_{A m}$ equal to the average value of $y_{A i}$ calculated by integrating over the whole tower. This assumption is not a serious error when the liquidphase resistance is predominant in the overall resisitance.

Under the above assumption, Eq. (1) and a part of the boundary conditions are changed as follows:

$$
\begin{gathered}
\frac{\partial \phi_{1}}{\partial \xi}=\frac{a_{G}}{\phi} \frac{\partial^{2} \phi_{1}}{\partial \zeta_{G}^{2}} \\
-\frac{P}{R T} \frac{\mathscr{D}_{G}}{Z_{G 0}} \frac{y_{A 0}-y_{A 0}^{*}}{\psi} \frac{\partial \phi_{1}}{\partial \zeta_{G}}=-\frac{\mathscr{D}_{A}\left(C_{A 0}^{*}-C_{A 0}\right)}{Z_{0}} \frac{\partial C_{A 1}}{\partial \zeta}
\end{gathered}
$$

\footnotetext{
$\dagger$ In the case of pure solute gas the value of $y_{A}$ is unity, that is, $y_{A m} \rightarrow 1$ and from Eq. (10) one obtains $\kappa_{1}=0$. Then Eq. (12) is rewritten as

$$
\bar{N}_{A}=2\left(C_{A 0}^{*}-C_{A 0}\right) \sqrt{\mathscr{D}_{A} / \pi t}
$$
}

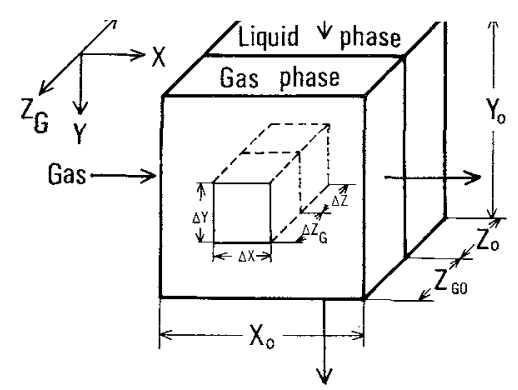

Fig. 1 Schematic model of crossflow absorption tower (wetted-wall type)

where

$$
\phi=1-y_{A m}
$$

By applying two dimensional Laplace transformation and the inverse transformation, the solutions of Eqs. (2) and (5) are given as

$$
\begin{aligned}
& C_{A 1}=\int_{0}^{\eta} \frac{\sqrt{\kappa_{1} \xi}}{\pi \sqrt{y\left(y+\kappa_{1} \xi\right)}} \operatorname{erfc}\left(\frac{\zeta}{2 \sqrt{a_{A}(\eta-y)}}\right) d y \\
& \phi_{1}=\int_{0}^{\xi} \frac{\sqrt{\eta / \kappa_{1}}}{\pi \sqrt{x\left(x+\eta / \kappa_{1}\right)}} \operatorname{erfc}\left\{\frac{\zeta_{G}}{2} \sqrt{\frac{\psi}{a_{G}(\xi-x)}}\right\} d x
\end{aligned}
$$

Here $\kappa_{1}$ in Eqs. (8) and (9) is defined by

$$
\kappa_{i}=\left(\frac{R T}{H}\right)^{2} \frac{\mathscr{D}_{A} t_{G} \phi}{\mathscr{D}_{G} t} \quad(i=1,2)
$$

The instantaneous absorption rate is given in the following form:

$$
N_{A}=-\left.\frac{\mathscr{D}_{A}}{Z_{0}} \frac{\partial C_{A}}{\partial \zeta}\right|_{\zeta=0}=\left(C_{A 0}^{*}-C_{A 0}\right) \sqrt{\frac{\mathscr{D}}{\pi t}} \frac{1}{\sqrt{\eta}+\kappa_{1} \xi}
$$

and the average absorption rate is

$$
\begin{aligned}
\bar{N}_{A}= & \int_{0}^{1} \int_{0}^{1} N_{A} d \xi d \eta / \int_{0}^{1} \int_{0}^{1} d \xi d \eta \\
= & 2\left(C_{\Delta 0}^{*}-C_{A 0}\right) \sqrt{\frac{\mathscr{D}_{A}}{\pi t}} \frac{2}{3}\left(\frac{1}{\sqrt{1+\kappa_{1}}+\sqrt{\kappa_{1}}}\right. \\
& \left.+\frac{1}{1+\sqrt{1+\kappa_{1}}}\right)
\end{aligned}
$$

and in addition, from Eqs. (8) and (9), the concentration profile at the gas-liquid interface is given by

$$
\frac{y_{A i}-y_{A 0}^{*}}{y_{A 0}-y_{A 0}^{*}}=\frac{C_{A i}-C_{A 0}}{C_{A 0}^{*}-C_{A 0}}=\frac{2}{\pi} \arctan \sqrt{\frac{\eta}{\kappa_{1} \xi}}
$$

From Eq. (13) and the boundary conditions, the function of concentration profile at the gas-liquid interface $f(\xi, \eta)$ is expressed by

$$
f(\xi, \eta)=\frac{2}{\pi} \arctan \sqrt{\frac{\eta}{\kappa_{1} \xi}}
$$

and $y_{A m}$ is expressed by:

$$
y_{A m}=\int_{0}^{1} \int_{0}^{1} y_{A i} d \xi d \eta / \int_{0}^{1} \int_{0}^{1} d \xi d \eta
$$

From Eqs. (13) and (15), the following equation is obtained: 
$A_{2}=\left(1 / \angle v \kappa_{1}-v \kappa_{1} / L-\pi / 4 \kappa_{1}\right)$

Then, from Eqs. (7), (10), (16) and (17), the value of $\kappa_{1}$ can be determined numerically by

$$
1-\left(\frac{H}{R T}\right)^{2} \frac{\mathscr{D}_{G} t}{\mathscr{D}_{A} t_{G}} \kappa_{1}=\frac{2\left(y_{A 0}-y_{A 0}^{*}\right)}{\pi}\left(A_{1}+A_{2}\right)+y_{A 0}^{*}
$$

\section{2 Irreversible second-order chemical reaction}

To solve this problem, on a basis similar to the reasoning mentioned above, the assumption proposed in the analysis of physical absorption is applied. In addition, it is assumed that the concentration of the absorbing liquid is replaced by the interfacial concentration $C_{B i}$, which is held constant by considering the average value over the whole tower. Under the above assumption, the basic differential equations for chemical absorption can be given in the following form:

$$
\begin{array}{ll}
\frac{\partial \phi_{2}}{\partial \xi}=\frac{a_{G}}{\phi} \frac{\partial^{2} \phi_{2}}{\partial \zeta_{G}^{2}} & \text { for gas phase } \\
\frac{\partial C_{A 2}}{\partial \eta}=a_{A} \frac{\partial^{2} C_{A^{2}}}{\partial \zeta^{2}}-b_{A} C_{A^{2}} & \text { for liquid phase }
\end{array}
$$

with the boundary conditions

$$
\begin{aligned}
& \text { at } \xi=0 \quad ; \quad \phi_{2}=0 \\
& \text { at } \eta=0 \quad ; \quad C_{A^{2}}=0 \\
& \text { at } \zeta_{G}=\zeta=0 ; \quad \phi_{2}=1-C_{A 2}, C_{A 2}=g(\xi, \eta) \text {, } \\
& -\frac{\mathscr{D}_{G}}{Z_{G 0}}-P \frac{y_{A 0}}{R T} \frac{\partial \phi_{2}}{\partial \zeta_{G}} \\
& =-\frac{\mathscr{D}_{A} C_{A 0}^{*}}{Z_{0}} \frac{\partial C_{A 2}}{\partial \zeta} \\
& \text { at } \zeta_{G} \rightarrow \infty \quad ; \quad \phi_{2}=0 \\
& \text { at } \zeta \rightarrow \infty \quad ; \quad C_{A 2}=0
\end{aligned}
$$

where

$$
b_{A}=k_{r} C_{B i} t, C_{A 2}=C_{A} / C_{A 0}^{*}, \phi_{2}=\left(y_{A 0}-y_{A}\right) / y_{A 0}
$$

The solutions of these equations are obtained easily by using the method of Laplace transformation:

$$
\begin{aligned}
C_{A 2}= & \frac{1}{2} \int_{0}^{\eta} \frac{e^{-b_{A}^{y}} \sqrt{\kappa_{2} \xi}}{\pi \sqrt{y\left(y+\kappa_{2} \xi\right)}}\left[e ^ { \zeta \sqrt { b } _ { A } a _ { A } } \operatorname { e r f c } \left\{\frac{\zeta}{2 \sqrt{a_{A}(\eta-y)}}\right.\right. \\
& \left.+\sqrt{b_{A}(\eta-y)}\right\}+e^{-\zeta \sqrt{b_{A} / a}} \operatorname{erfc}\left\{\frac{\zeta}{2 \sqrt{a_{A}(\eta-y)}}\right. \\
& \left.\left.-\sqrt{b_{A}(\eta-y)}\right\}\right] d y \\
\phi_{2}= & \operatorname{erfc}\left(\frac{\zeta_{G}}{2} \sqrt{\frac{\psi}{a_{G} \xi}}\right)-F_{1}\left(\xi, \zeta_{G}\right) \\
+ & \frac{1}{2} \operatorname{erfc} \sqrt{b_{A} \eta}\left\{F_{1}\left(\xi, \zeta_{G}\right)-F_{2}\left(\xi, \zeta_{G}\right)\right\}
\end{aligned}
$$

${ }^{\dagger}$ In the case of pure solute gas, the following equation is obtained:

$$
\bar{N}_{A}^{*}=C_{A 0}^{*} \sqrt{\frac{\mathscr{D}_{A}^{-}}{t}}\left\{\left(\sqrt{b_{A}}+\frac{1}{2 \sqrt{b_{A}}}\right) \operatorname{erf} \sqrt{b_{A}}+\frac{1}{\sqrt{\pi}} e^{-b_{A}}\right\}
$$

where

$$
\left.\begin{array}{rl}
F_{1}\left(\xi, \zeta_{G}\right)= & \exp \left(b_{A} \kappa_{2} \xi+\zeta_{G} \sqrt{b_{A} \kappa_{2} \psi / a_{G}}\right) \\
& \times \operatorname{erfc}\left(\zeta_{G} \sqrt{\left.\psi / a_{G} \xi / 2+\sqrt{b_{A} \kappa_{2} \xi}\right)}\right. \\
F_{2}\left(\xi, \zeta_{G}\right)= & \exp \left(b_{A} \kappa_{2} \xi-\zeta_{G} \sqrt{b_{A} \kappa_{2} \psi / a_{G}}\right) \\
& \times \operatorname{erfc}\left(\zeta_{G} \sqrt{\phi / a_{G} \xi / 2-\sqrt{b_{A} \kappa_{2} \xi}}\right)
\end{array}\right\}
$$

and $\kappa_{2}$ is the positive root of Eq. (32).

As a result, the instantaneous absorption rate is

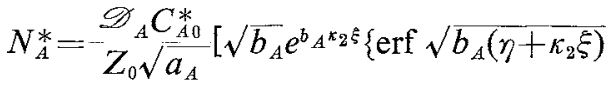

$$
\begin{aligned}
& \left.\left.\left.-\operatorname{erf} \sqrt{b_{A} \kappa_{2} \xi}\right\}+e^{-b} A^{\eta} / \sqrt{\pi(\eta+}+\kappa_{2} \xi\right)\right]
\end{aligned}
$$

and the average absorption rate is

$$
\begin{aligned}
\bar{N}_{A}^{*}= & C_{A 0}^{*} \sqrt{\mathscr{D}_{A} / t}\left[\{ ( 1 - 1 / 2 b _ { A } ) / \kappa _ { 2 } \} \left\{F_{3}\left(b_{A}\right)\right.\right. \\
& \left.-\operatorname{erf} \sqrt{b_{A}} / \sqrt{b_{A}}+2 \sqrt{\kappa_{2} / \pi}\right\}+F_{3}\left(b_{A}\right) \\
& \left.+e^{-b_{A}} /\left\{b_{A} \sqrt{\pi}\left(1+\sqrt{1+\kappa_{2}}\right)\right\}\right]
\end{aligned}
$$

where

$$
F_{3}\left(b_{A}\right)=\left\{e^{b_{A} \kappa_{2}} / \sqrt{b_{A}}\right\}\left\{\operatorname{erf} \sqrt{ } b_{A}\left(1+\kappa_{2}\right)-\operatorname{erf} \sqrt{ } b_{A} \kappa_{2}\right\}
$$

From Eqs. (22) through (25), the concentration profile at the gas-liquid interface is given by

$$
\frac{y_{A i}}{y_{A 0}}=\frac{C_{A i}}{C_{A 0}}=\frac{2}{\pi} \int_{0}^{\sqrt{\eta / \kappa_{2} \xi}} \cdot \frac{e^{-b} A^{k_{2} \xi t^{2}}}{1+t^{2}} d t
$$

and $g(\xi, \eta)$ in the boundary conditions is expressed by the following equation.

$$
g(\xi, \eta)=\frac{2}{\pi} \int_{0}^{\sqrt{\eta / \kappa_{2} \xi}} \frac{e^{-b} A^{k_{2} \xi t^{2}}}{1+t^{2}} d t
$$

From Eqs. (29) and (30), $y_{A m}$ at the gas-liquid interface is as follows:

$$
\frac{y_{A m}}{y_{A 0}}=\int_{0}^{1}\left(1+\kappa_{2} \xi\right) g(\xi, 1) d \xi-\frac{2}{3} \sqrt{\frac{\kappa_{2}}{\pi b_{A}}} \operatorname{erf} \sqrt{b_{A}}
$$

and $\kappa_{2}$ is expressed by

$$
\begin{aligned}
1-\left(\frac{H}{R T}\right)^{2} \frac{\mathscr{D}_{G} t}{\mathscr{D}_{A} t_{G}} \kappa_{2}= & y_{A 0}\left\{\int_{0}^{1}\left(1+\kappa_{2} \xi\right) g(\xi, 1) d \xi\right. \\
& \left.-\frac{2}{3} \sqrt{\frac{\kappa_{2}}{\pi b_{A}}} \operatorname{erf} \sqrt{b_{A}}\right\}
\end{aligned}
$$

The integration terms Eqs. (29) to (32) are not solved analytically. In this paper, the terms are numerically calculated by the use of Gauss-Legengre integration formula.

1. 3 Relation between overall mass transfer coefficient and individual mass transfer coefficient

In the case of gas mixture, the overall mass transfer coefficient is expressed by the following equation according to the film theory

$$
\bar{N}_{A}=K_{G}\left(p_{A 0}-p_{A 0}^{*}\right)=K_{L}\left(C_{A 0}^{*}-C_{A 0}\right)
$$

By comparing Eq. (33) with Eqs. (12) or (27) at $C_{A 0}=0$, one obtains 
for physical absorption

$$
\begin{aligned}
K_{L}= & \sqrt{\mathscr{D}_{A} / t}\left[\{ ( 1 - 1 / 2 b _ { A } ) / \kappa _ { 2 } \} \left\{F_{3}\left(b_{A}\right)-\operatorname{erf} \sqrt{b_{A}} / \sqrt{b_{A}}\right.\right. \\
& \left.\left.+2 \sqrt{\kappa_{2} / \pi}\right\}+F_{3}\left(b_{A}\right)+e^{-b_{A}} /\left\{b_{A} \sqrt{\pi}\left(1+\sqrt{1+\kappa_{2}}\right)\right\}\right]
\end{aligned}
$$

for irreversible second-order reaction

When Henry's law is valid, the relation between $K_{L}$ and $K_{G}$ is expressed by:

$$
K_{G}=K_{L} / H
$$

and the value of $K_{G}$ is calculated by substituting Eqs. (34) or (35) into Eq. (36).

Now, let us discuss individual mass transfer coefficients, $k_{L}$ and $k_{G}$. By the definition of film theory

$$
\bar{N}_{A}=k_{G}\left(p_{A 0}-p_{A i}\right)=k_{L}\left(C_{A i}-C_{A 0}\right)
$$

For gas mixture, the interfacial concentration is not kept constant in a tower. If one uses average values over the whole tower in place of $p_{A i}$ and $C_{A i}$ and compares Eq. (33) with Eq. (37), then the following relationships are obtained:

$$
\begin{aligned}
K_{G} / k_{G} & =1-\left(y_{A m}-y_{A 0}^{*}\right) /\left(y_{A 0}-y_{A 0}^{*}\right) \\
K_{L} / k_{L} & =\left(C_{A m}-C_{A 0}\right) /\left(C_{A 0}^{*}-C_{A 0}\right) \\
& =\left(y_{A m}-y_{A 0}^{*}\right) /\left(y_{A 0}-y_{A 0}^{*}\right)
\end{aligned}
$$

\section{Discussion}

Here, let us take some examples for $\mathrm{CO}_{2}-\mathrm{N}_{2}$ gas mixture- $\mathrm{NaOH}$ system. Figure 2 compares the theoretical results calculated by the solution in this paper with those in a previous paper ${ }^{2}$ for $20 \% \mathrm{CO}_{2}$ and $30 \%$ $\mathrm{CO}_{2}$ in gas mixture. Solid lines represent the former and broken lines the latter. As can be seen from this figure, a difference in absorption rate between the cases of taking into account and not taking into account the gas-phase resistance increases with increasing normality of $\mathrm{NaOH}$ aqueous solution or with decreasing composition of the solute gas. For second-order chemical reactions, this difference is caused by the discrepancy between $C_{A}$ and $C_{A}^{*}$ at the gas-liquid interface ${ }^{1)}$, and in addition, when the time of contact between phases increases, that is, when the reaction lies in the region of instantaneous reaction, as is evident from Eq. (10). the values of $\kappa_{1}$ and $\kappa_{2}$ become 0 , and then no difference in the absorption rate between the two cases mentioned above is observed.

Figure 3 shows the relationship between $\vec{N}_{A}^{*}$ and $t$ for chemical absorption as a parameter of $t_{G}$. These theoretical lines are calculated by Eqs. (27), (28) and (32), and when $t_{G}=0$ the lines indicate the results in the case of taking into account only the liquid-phase resistance. For the purpose of comparison, the relation $\bar{N}_{A}$ vs. $t$ for physical absorption calculated by Eqs. (12) and (18) is illustrated as a broken line. As is evident from this figure, for physical absorption the

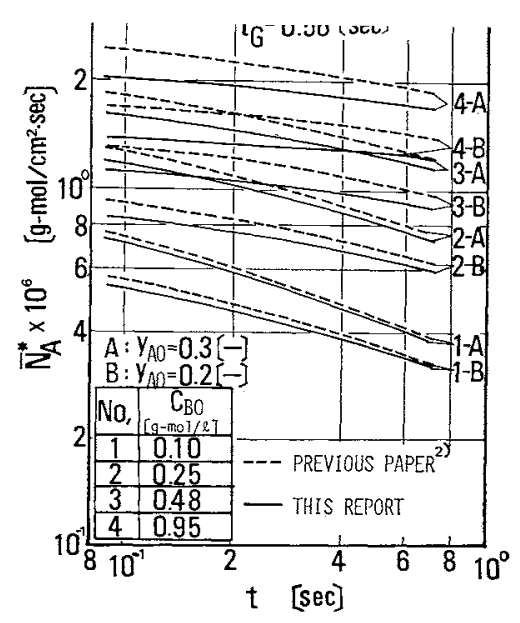

Fig. 2 Comparison of the theoretical results of $\bar{N}_{A}^{*}$ at $20^{\circ} \mathrm{C}$

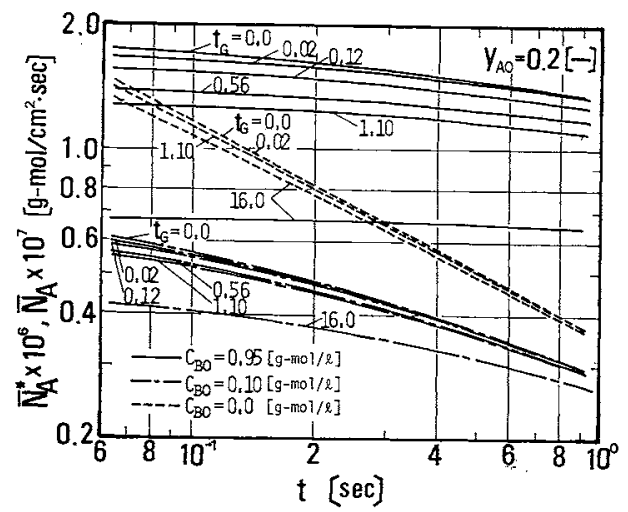

Fig. 3 Effect of gas flow rate on the average absorption rate at $20^{\circ} \mathrm{C}$ for gas absorption with and without reaction ( $\mathrm{NaOH}-\mathrm{CO}_{2}$ system)

effect of gas flow rate on average absorption rate is scarcely observed in the region below $t_{G}=1.1$. That is, as the solute gas used in this example is carbon dioxide, the absorption rate is relatively small, and even if the gas flow rate is small the change in interfacial concentration of the solute gas is not so large that this tendency is observed. On the other hand, for chemical absorption the effect of gas flow rate on average absorption rate is greater than that for physical absorption. In particular, when the normality of $\mathrm{NaOH}$ aqueous solution is large, its effect is considerable. In addition, a difference in absorption rate between the cases of taking into account and not taking into account the gas-phase resistance increases with increasing the value of $t_{G}$. That is, when $t_{G}=0.02$ and the normality of $\mathrm{NaOH}$ aqueous solution is $0.95 \mathrm{~N}$, its difference is $4 \%$ at most and for $t_{G}=16.0$ and $C_{B 0}=0.95 \mathrm{~N}$, it is about $40-50 \%$. As the gas flow rate decreases, the change in interfacial concentration of the solute gas becomes larger and the tendency mensioned above is observed. 


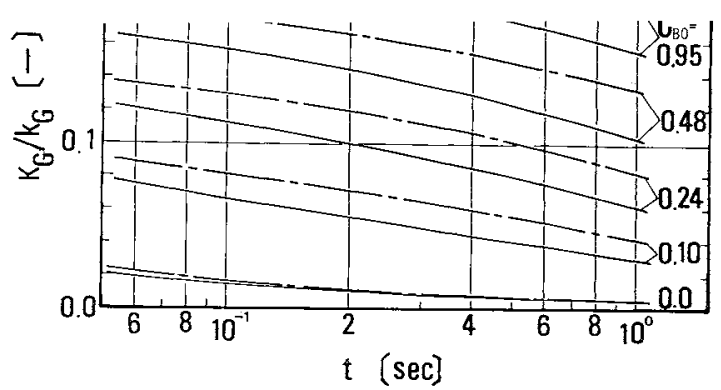

Fig. 4 Relations between $K_{G} / k_{G}$ and $t$ for $\mathrm{CO}_{2-}$ $\mathrm{NaOH}$ solutions at $20^{\circ} \mathrm{C}$ where $t_{G}=0.56 \mathrm{sec}$

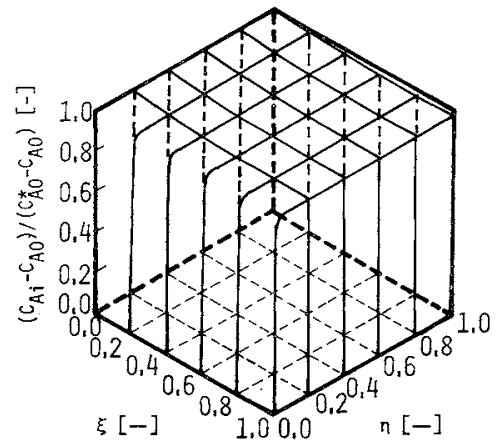

Fig. 5 Concentration profile of solute gas at the gas-liquid interface for physical absorption of carbon dioxide into water: $t_{G}=0.56 \mathrm{sec}, t=0.2 \mathrm{sec}$, $y_{A 0}=0.2$ mole fraction

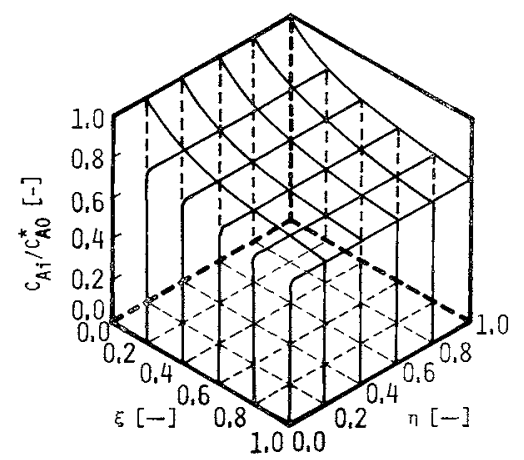

Fig. 6 Concentration profile of solute gas at the gas-liquid interface for chemical absorption of carbon dioxide into $\mathrm{NaOH}$ aqueous solution: $t_{G}=$ $0.56 \mathrm{sec}, t=0.2 \mathrm{sec}, y_{A 0}=0.2$ mole fraction, $C_{B 0}=$ $0.95 \mathrm{~g}-\mathrm{mol} / \mathrm{l}$

In Fig. 4, $K_{G} / k_{G}$ is plotted against $t$ with a parameter of the normality of $\mathrm{NaOH}$ aqueous solution for $y_{A 0}=0.2$ and 0.3. As is obvious from this figure, the $K_{G} / k_{G}$ value increases with increasing normality of $\mathrm{NaOH}$ solution, that is, the gas-phase resistance increases in proportion to the normality of $\mathrm{NaOH}$ aqueous solution. When its normality is $0.95 \mathrm{~N}$, the ratio of the gas-phase resistance to overal resistance reaches values above $20 \%$ under certain circumstances. concentration for physical absorption of carbon dioxide into water and for chemical absorption of carbon dioxide into $\mathrm{NaOH}$ aqueous solutions, respectively. As is evident from these figures, it is found that the change in interfacial concentration for chemical absorption is larger than that for physical absorption.

As a result of these theoretical considerations, when a solute gas of relatively small solubility is used in industrial processes, for physical absorption the gasphase resistance may be negligible and for chemical absorption a significant concentration change appears in the solute gas at the gas-liquid interface. In the design of contacting equipment for absorptions accompanied by second-order reactions, the absorption rate and the equilibrium concentration at the gasliquid interface should be researched in detail by using the solutions in this paper.

\section{Conclusion}

An analytical solution for absorption accompanied by a irreversible second-order reaction in a crossflow gas-liquid contactor was studied by considering both gas- and liquid-phase resistances to absorption and was compared with the solution in the previous paper, in which it was assumed that the gas-phase resistance to absorption was negligible. In a system of relatively small solubility, such as the gas mixture of $\mathrm{CO}_{2}$ and air$\mathrm{H}_{2} \mathrm{O}$ system, change in interfacial concentration of the solute gas is scarcely observed, and consequently the gas-phase resistance is negligible for the analysis of physical absorption. On the other hand, for absorptions with irreversible second-order reactions by the use of above solute gas, for instance, the gas mixture of $\mathrm{CO}_{2}$ and air- $\mathrm{NaOH}$ system, a significant concentration change in the solute gas at the gas-liquid interface observed. In the design of contacting equipment for absorptions accompanied by second-order reactions, the absorption rate and the profile of interfacial concentration in a tower should be studied in detail by using the solutions in this paper.

\section{Nomenclature}

$A_{1}, A_{2} \quad=$ defined by Eq. (17) [-]

$a_{A}, a_{C} \quad=$ dimensionless variables $\left(=\mathscr{O}_{A} t / Z_{0}^{2}, \mathscr{D}_{G} t_{Q} / Z_{G 0}^{2}\right)$

$b_{A} \quad=$ dimensionless variable $\left(=k_{r} C_{B i} t\right) \quad[-]$

$C_{A}=$ concentration of solute gas in liquid phase

$C_{A}^{*}=$ concentration of solute gas in liquid phase in equilibrium with $y_{A} \quad\left[\mathrm{~g}-\mathrm{mol} / \mathrm{cm}^{3}\right]$

$C_{B} \quad=$ concentration of reactant in liquid phase

$C_{A 1}, C_{A 2}=$ dimensionless concentrations of solute gas in 
$F_{1}\left(\xi, \zeta_{G}\right), F_{2}\left(\xi, \zeta_{G}\right)=$ defined by Eq. $(25)$

$F_{3}\left(b_{A}\right) \quad=$ defined by Eq. (28)

$f(\xi, \eta), g(\xi, \eta)=$ functions of concentration profile at gasliquid interface for physical absorption and for chemical absorption, respectively $\quad[-]$

$\begin{array}{lll}H & =\text { Henry's constant } \quad\left[\mathrm{atm} \cdot \mathrm{cm}^{3} / \mathrm{g}-\mathrm{mol}\right] \\ K_{G} & =\text { overall mass transfer coefficient in gas phase } \\ & & {\left[\mathrm{g}-\mathrm{mol} / \mathrm{cm}^{2} \cdot \mathrm{atm} \cdot \mathrm{sec}\right]}\end{array}$

$K_{L} \quad=$ overall mass transfer coefficient in liquid phase [ $\mathrm{cm} / \mathrm{sec}]$

$k_{G} \quad=$ gas-film mass transfer coefficient

$\left[\mathrm{g}-\mathrm{mol} / \mathrm{cm}^{2} \cdot \mathrm{atm} \cdot \mathrm{sec}\right]$

$k_{L} \quad=$ liquid-film mass transfer coefficient $\quad[\mathrm{cm} / \mathrm{sec}]$

$k_{r} \quad=$ second-order reaction rate constant

$\left.\left[\mathrm{cm}^{3} / \mathrm{g}-\mathrm{mo}\right] \cdot \mathrm{sec}\right]$

$N^{*}, N \quad=$ instantaneous absorption rates with and without chemical reaction, respectively $\left[\mathrm{g}-\mathrm{mol} / \mathrm{cm}^{2} \cdot \mathrm{sec}\right]$

$\bar{N}^{*}, \bar{N} \quad=$ average absorption rates with and without chemical reaction, respectively $\left[\mathrm{g}-\mathrm{mol} / \mathrm{cm}^{2} \cdot \mathrm{sec}\right]$

$P \quad=$ total pressure in gas phase [atm]

$p \quad=$ partial pressure in gas phase $\quad[\mathrm{atm}]$

$R \quad=$ gas constant $\quad\left[\mathrm{cm}^{3} \cdot \mathrm{atm} / \mathrm{g}-\mathrm{mol} \cdot{ }^{\circ} \mathrm{K}\right]$

$\begin{array}{lll}T & =\text { temperature } \quad\left[{ }^{\circ} \mathrm{K}\right],\left[{ }^{\circ} \mathrm{C}\right]\end{array}$

$t \quad=$ contact time between gas and liquid

$t_{G} \quad=$ passing time of gas through a tower

$V_{G} \quad=$ average velocity of gas

$V_{\max } \quad=$ maximum velocity of liquid film

$X \quad=$ direction of gas flow

$X_{0} \quad=$ depth of tower

$Y \quad=$ direction of liquid flow

$Y_{0} \quad=$ height of tower [sec]

[sec]

$[\mathrm{cm} / \mathrm{sec}]$

$[\mathrm{cm} / \mathrm{sec}]$

[cm]

[cm]

[cm]

$[\mathrm{cm}]$
$Z, Z_{G} \quad=$ distances into liquid phase and into gas phase

$Z_{c 0}, Z_{0}=$ thichnesses of gas layer and liquid film, respectively

$\zeta, \zeta_{G}, \eta, \xi=$ dimensionless lengths $\left(=Z / Z_{0}, Z / Z_{G 0}, Y / Y_{0}, X / X_{0}\right)$

$\kappa_{1}, \kappa_{2}=$ dimensionless parameters defined by Eq. (10)

$\phi_{1}, \phi_{2} \quad=$ dimensionless concentrations of solute gas in gas phase $\left(=\left(y_{A 0}-y_{A}\right) /\left(y_{A 0}-y_{A 0}^{*}\right),\left(y_{A 0}-y_{A}\right) / y_{A 0}\right) \quad[-]$

$\phi \quad=$ dimensionless variable defined by Eq. (7) $\quad[-]$

〈Subscripts〉

$\begin{array}{ll}A & =\text { solute gas } \\ B & =\text { reactant in }\end{array}$

$B=\quad=$ reactant in liquid phase

$G \quad=$ gas phase

$i \quad=$ gas-liquid interface

$m \quad=$ average at gas-liquid interface

$0 \quad=$ inlet

\section{Literature Cited}

1) Emmert, R. E. and R. L. Pigford: AIChE J., 8, 171 (1962).

2) Hayashi, Y., E. Hirai and N. Shimizu: Kagaku Kogaku Ronbunshu, 1, 408 (1975).

3) Hayashi, Y., E. Hirai and N. Shimizu: J. Chem. Eng. Japan, 9, 198 (1976).

(A part of this paper was presented at Hokuriku Meeting of The Soc. of Chem. Engrs., Japan, at Toyama, July, 1976.) 